\title{
Sachregister zu Band 93
}

Die fett gedrackten Zahlen bedeuten Eigenarten. Bb. = Buchbesprechungen.

A Ablatio retinae 349

nach indirekter Verletzung 247

Makulaloch bei der -104

mit Lochbildung am hinteren Au-genpol, geheilt durch diathermische Stichelung 101, 261

operative Behandlung der - 172

modifizierte Lochbrille in der Re-konvaleszenz bei - 254

Ach $\tau$ omasie 240

Adiesche $\tau$ Symptomenkomplex 95

Aegyptische augenärztl. Gesellschaft

259 (Bb.) Alkohol, Schädigung des Sehnerven

durch - 304 Alopezie, isolierte - der Zilien 98 Aneurysmen der Carotis interna, Seh-

störungen bei - 319 Angiomatosis retinae et cerebeili 325 Angiopathia traumatíca nach Schädeltrauma 327 Aniseikonie, Brillen für - 357 Anisometrie, Brillen für - 357 Arachnitis

opticochíasmatíca 309 Arachnodaktylie u. Linsenektopie 74

- $\quad$ mit kongenitalem Uvea-Linsen-kolobom 163

Arcus lipoides, partieller - bei lim-busnahen tumorösen Prozessen der Konjunktiva 197

Astigmatismus, Korrektur des hoch-gradigen - 160

AT 10243

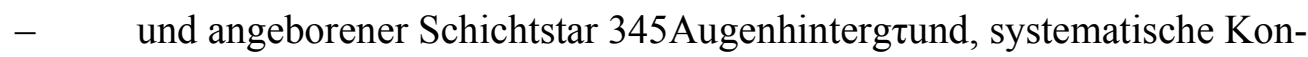

trolle des - bei Hypertonikern 334

- gefäßähnliche Streifen am - 238

- $\quad$ Blutungen des - bei leptomenin-gitischen Spontanblutungen 320

Augenmigräne 243, 319 Augenmuskeln 144 B Bazillen, die durch bipolare - ver-

ursachten Bindehautaffektionen 57 BengaiГotfärbung b. Konjunktivitis 251 Besnier-Boecksche

Krankheit, Augen-

veränderungen bei - 257 Bewegungsapparat d. Auges 144 (Ber.) Bienengift, Behandlung der

Iritis

rheumatica mit - 365 Bindehaut s. Conjunctiva Blepharospasmusbrille 235 Blícklähmungen 153

Blutdruck in den Netzhautgefäßen 167

Blutdruck u. Netzhautarteriendruck 334

Boecksche Krankheit, Augenverände-rungen bei - 236, 257

Brillen für Anisometropie und Aniseikonie 357

Brillen-Dermatitis 366

Brillengeråt, orthopädisches - für das Kleinkind 253

Brustdrüsenkrebs mit Metastasen in der Ade $1 / 8$ aut 95, 104

Bürgersche Krankheit 108

C Chalkosis der Linse 76 Charlinscher Symptomenkomplex 92 Chevalier Christiaen 94 Chiasma 309,310 
- $\quad$ Herd im - bei multipler Sklerose237

Chiasmasyndrom nach Schädelver-verletzung 104

- $\quad$ in der Schwangerschaft 115Choríoidea, metastatisches Karzinom

der - bei Brustdrüsenkrebs 95, 104

Riesentuberkel der -102

Pseudotumor der - Ill Conjunctiva, die durch bipolare Bazillen verursachten Affektionen der $-\quad 57$

diphtherische Erkrankung der - 362

Teratom der -164

- $\quad$ Salbenfüllung des - sackes 49Conjunctivitis, Bengalrotfärbung bei

- 251

Cornea, seltene Erkrankung der - 243

primäre bandförmige Degeneration der - 164

walzenförmige Vorwölbung und Verdünnung der unteren Fläche der - 164

partieller Arcus lipoides bei lim-busnahen tumorösen Prozessen der

- $\quad 197$

- $\quad$ Blasenbildung der - bei Boeck-schem Sarkoid 236

Cushingsche Krankheit 324 Cutireaktion der Lues 186 Cysticercus cellulosae 336

$\mathrm{D}$

Devicsche Krankheit 316 Desensibilisierungsbehandlung 171 Diabetes insipidus nach

Schädelver-letzung 104

Sachregister zu Band 93

369

Dathermie. Ablatio retinae mit Loch-

bildung geheilt durch diathermische

Stichelung 101, 261 Dinit okörper, Katarakt nach An-

wendung von - 75 Diphtherie, diphth. Erkrankung der

Bindehaut 362 Dmckschwankungen nach Vorlage-

rungen 175 Dynamometrie d. Netzhautgefäße 333 Dysostosis cranio-facialis 90

E Eisensplitterverletzungen 353 Endangiitis oblíterans 320 Enophthalmus, einseitiger

angeborener

- 85.Enzephalitis 155

- $\quad$ epidemica 317

Enzephalomyelitis disseminata, Neuritis optica bei -316

Exophthalmus 85, 86, 87

- $\quad$ einseitiger 255

F Faserve $\tau$ lauf in den Sehbahnen 311 Feb $\tau$ is uveo-parotidea 160 Filmvorfüh $\pi m g e n ~ v$.

Operationen 349 Fusion 146

G Gefäßähnliche Streifen am Augen-

hintergrund 238 Gefäßbildungen und Gefäßgeschwülste

des Gehirns 325 Gehirnabszeß 318 Gehirngeschwülste 320 Gehirnsklerose, tuberkulöse 328

Gelbkreuzgasvergiftung, Spätschädi-

gung der Augen nach - 252 Gesichtsfeld, Leistung des peripheren

- $\quad$ nach der Staroperation 242Gesichtsfelddefekte, Asymmetrie der

- bei homonymer Hemianopsie 345 Gewebsbildungen, ungewöhnliche - 
an den Papillen 235, 239 Glaskörper 82 (Bericht)

- $\quad$ ein neues Phänomen am - 158GlasköГperfistel, Anlegung einer -

117

Glaskörpergrenzmembranen, Abdrän-

gung der - 121 Glaukom und Naevus flammeus 105

Kapsel-G. 78

und Stoffwechsel 107 Glaukomoperatíonen 353

Extraktion der klaren Linse ale

- $\quad$ 174Gliome 362

- $\quad$ der Netzhaut 349, 352Ganioskopische Demonstrationen 158Gradenigosche Krankheit

154

$\mathrm{H}$

Haemangioma cavemosum orbitae,

Lymphfollikel im - 104 Hemeralopie 241

Hemianopísche Halluzinationen 311 Hemianopsie, binasale 348

- $\quad$ Asymmetrie d. Gesichtsfelddefektebei angeborener - 345

Herpes, endogener $-\mathrm{u}$. Trauma 176 Herpes zoster, Augenmuskelstörungen

nach -317 Heterochromie m. Status dysraphicus

327 HeteГophoríe 147 Homòopathische Arzneimittel 240 Homhaut s. Cornea Hypertonie, systematische Kontrollen

des Augenhintergrundes bei - 334 Hypophysengeschwiilste, Sehstörun-

gen bei -- 323 Hypopyoniritis, rezidivierende 175 Hypotonie des Auges, Stauungspapille

bei - 133 Hypovitaminose. C-Hyp. und Alters-

star 343

I

Idiotie, familiäre amaurotische 174 lnversio (recessio) íridis durch spate Nachblutung nach

Kontusion 222 Iridectomia ab externo 1 Iridotomie bei der Staroperation 93 Iridotorsion 176

Iris, Rubeosis iridis diabetica 162 Irisatrophie 162, 163

- $\quad$ ein operatives Kolobom vortäu-schende - 94

Irisknötchen b. Neurofibromatose 137

- $\quad$ bei Boeckschem Sarkoid 236, 257Iristumor, symmetrischer 163Iriszerreißungen,

Mechanik der - 240Iriszyste, operierte 96

$/$ ríf $^{1} / 8$, Zustandekommen der ektogenen -252

- $\quad$ Behandlung der rheumatischen -mit Bienengift 365

K Kapselglaukom 78 Katarakt 72 ff.

myotonische 164

C-Hypovitaminose und Altersstar 343

bei Neurodermie 106

AT 10 und angeborener Schicht-star 345

Kataraktoperationen 78, 345

intrakapsulãre 103, 173, 244, 246

Ausziehung des Altersstars mit dem Häkchen 65, 346

Iridotomie bei der -93

370

Sachregister zu Band 93 
Kataraktoperatíonen, Leistung d. peri-pheren Gesichtsfeldes n. der - 242

- $\quad$ Irisatrophie imAnschluß an - 163Keilbeinflügel, Meningiom des kleinen

- $\quad$ 324Keratins ulce

357 Kolobome des Opticus 165 Kontusion, totale Inversio iridis durch

spate Nachblutung nach - 222 Konveigenz 146 Krebs 350

- $\quad$ metastasen im Auge 278

$\mathrm{L}$

Leptomeningitische Spontanblutungen,

Augenhintergrundsblutungen bei -

320 Lichtreflex, Störungen des - bei den

syph. Erkrankungen des Nerven.

systems $180(\mathrm{Bb}$.$) Líder, Operation des angeborenen$

Koloboms des - 345 Lidtumoren, metastatische 31 Linse 69 (Bericht)

- Biologie der - 347Lochbrille, modifizierte - in der Re-

konvaleszenz bei Netzhautablösung 254 Luftfahrtmedizin, das Sehorgan in der

$-\quad 258$

Lymphfollikel im Hämangioma caver-nosum der Orbita 104

$\mathrm{M}$

Makulaloch bei d. Ablatio retinae 104

- $\quad$ paramakulares - nach Tennis-verletzung 161

Marcus Gunnsches Phãnomen 159 Meningiome 324 Meningitis cerebrospinalis 318

Mesothorium, Wirkung des - auf

das Auge 361 Migtâne, Augen-M. 243, 319 Mikrokomea mit hoher Myopie 97 Mißbildungen

105 Mumps-Neuritis 302 Myasthenie 154 Myopathien, Augenstörungen bei

327 Myopie, hohe - mit Mikrokomea 97 Myotonische Katarakt 164

N Nachstaroperation 335 Naevus flammeus und Glaukom 105 Natriumdampflampe 362

Naturheilkunde 105 Nebenhöhlen 90

Nekrosen in sympathisierenden In-

filtraten 293 Nervenkrankheíten u. Auge 314 (Ber.) Nervus oculomotorius, rezidivierende

Parese des -355 Nervus opticus 300 (Bericht)

Kolobome des -165

atrophie, Behandlung der tabi-schen -305

Netzhaut s. Retinae

Neuritis retrobulbaris 104,300

- $\quad$ in der Schwangerschaft 115

- $\quad$ bei lateraler Sinusitis 92 Neurodermie, Katarakt bei - 106Neurofibromatose, Beteiligung

der

Augen bei - 137 Nystagmus 225 (Bericht)

- retractorius 104

$\mathrm{O}$

Oedembereitschaft 350 Operationen, Filmvorführungen von

-349 Orbita 85 (Bericht)

$\mathrm{P}$

Papule, ungewöhnliche Gewebsbildun-

gen an den - 235, 239 Papillenödem, Prognose beim - 177 Parinaudsche Krankheit 52, 55

Pemphigus des Auges 349 Periphlebitis retinalis 341 
- $\quad$ sympathicus 293

- $\quad$ Unterschied zwischen Thromb-angitis obliterans retinae und - 35

Pigmentdegeneration der Netzhaut, Sympathektomie bei - 168

Plasmom, Entstehung des - 181

Plazentarblut, intramuskuläre Ein-spritzung von - bei Sehnerven-atrophie 306

Pseudotumor orbitae 109, Ill

Pupillotonie 327, 339

$\mathrm{R}$

Reflexbilder des Auges, Stereophoto-

grammetrie der - 237 Retina, ausgedehnte periphere zystoide

Degeneration der - 99

- $\quad$ Sympathektomie bei tabischerAtrophie der - 168

Retinagefäße, Durchmesser der-114

Messung des Blutdrucks in den - 167

Dynamometrie der - 333

Druck in den Arteríen und allge-meiner Blutdruck 334

Röntgenbehandlúng d. Thrombose der Venen 170

Retinitis serosa Ill

Sachregister zu Band 93

371

Retraktion 154

Röntgenaufnahme der Augen, skelett-

freie - 104 Röntgenbehandlung der Thrombose

der Netzhautvenen 170 Ròntgenstar 76 Rosacea-Keratítis 352 Rubeosis iridis diabetica 162

S Safáfsche Ringlinie 100 Salbenfüllung des Bindehautsackes 49 Sarkoid Boeck, Knötcheniritis und Blasenbildung der Hornhaut bei

- $\quad$ 236, 257Schädel 90 (Bericht)Schädelgrube, Differentialdiagnose bei

Erkrankungen der vorderen - 347 Schädelverletzung, Chiasmasyndrom

und Diabetes insipidus nach - 104 Schiefhals 249, 153 Schielen 148

- $\quad$ chirurgischer Eingriff bei para-lytischem - 340

Schildersche K $\tau$ ankheit 317 Schille $\Gamma$-Christian-Handsche Krankheit

112 SchmeГzproblem 103 Schwangerschaft, Chiasmasyndrom

und Neuritis retrobulbaris in der

- $\quad$ 115Sehhilfen, frühe 338Sehnerv s. Nervus opticusSehrinde 311

Seminom m. Fundusveränderungen 166 Sinusitis, Neuritis retrobulbaris bei

latenter - 92 Skleromalazie 364 Sklerose, multiple - und Auge 315

- $\quad$ Chiasmaherd bei - 237Spaltlampenuntersuchungen 244Spastolypt 243

Status dysraphicus mit Heterochromie

327 Stauungspapule 307, 308

- $\quad$ Differentialdiagnose zwischen ech-ter und Pseudo- - 110

- $\quad$ bei Hypotonie des Auges 133Stereophotogrammetrie der Reflex-

bilder des Auges 237 Stoffwechsel und Glaukom 107 Strahlenkörper, Verhalten des - nach

Zyklodialyse 248 Strumektomie, Tetanie nach - 349 Sympathektomie bei tabischer Atro-

phie und Pigmentdegeneration der

Netzhaut 168 Sympathisierende Infiltrate, Nekroseh 
in - 293 Synkinesien, okulo-pulpebrale 340

Syphilis 314

Cutireaktion der -186

Störungen des Lichtreflexes der Pupille bei den syph. Erkrankungen des Nervensystems 180

(Bb.)

$\mathrm{T}$

Tabak, Schädigung des Sehnerven

durch - 304 Tennisballverletzung mit paramaku-

larem Loch 161 Teratom der Conjunctiva 164 Tetanie und Katarakt 73

- $\quad$ nach Strumektomie 349Thrombangitis obliterans retinae, Un-

terschied zwischen Periphlebitis retinalis und -35

Thrombose der Netzhautvenen, Ròntgenbehandlung bei - 170

Trachom, Uebertragu $\pi$ gsversuche bei

- 253

Tuberkel, Riesen - der Aderhaut 102 Tuberkulid der Makulagegend Ill Tuberkulose des Auges

342

- $\quad$ serologische Diagnostik der Augen

$-\quad 252$

Tumor, retrobulbärer - 160

- $\quad$ operative Behandlung der intra-okularen - 113

$\mathrm{U}$

Ultrakurzwellen, Einwirkung der - auf das gesunde Auge 355

Ultraviolette Strahlen, Linsenschädi-gungen durch - 76

Uvea-Linsenkolobom, Arachmodak-tylie bei kongenitalem - 163

$\mathrm{V}$

Veråtzungen 252, 359

Vertikallesen 254

Vitamin A im Blut und Keratomalazie

357 Vitamin C, Bedeutung des - für den

Linsenstoffwechsel $70 \quad$ Stoffwechsel des Auges 244

- Hypovitaminose und Altersstar 343Vorderhirn und Auge 360Vorderkammerzyste, postoperative 165Vorlagerungen, Druckschwankungen

nach -175

W Wimpern, Augenschädigungen durch

F. Färben der - 99 Z Ziliarkörper

Sarkom des - 97 Zilien

isolierte Alopezie der - 98 Zirbeldrüse

Neubildungen der - 326 Zyklodialyse

Verhalten des Strahlen-

körpers nach -248 